ISSN 0103-8478

\title{
A Catuama e o bilobalide na regeneração nervosa periférica de ratos submetidos à secção do nervo isquiático
}

\section{Catuama and bilobalide on peripheral nerve regeneration in rats following sciatic nerve section}

\author{
Rodrigo Norberto PereiraI Nádia Delistoianov ${ }^{\text {II }}$ João Henrique Perotta ${ }^{\text {II }}$

 \\ Antonio Carlos Alessivi
}

RESUMO

\begin{abstract}
A Catuama $^{\circledR}$ é a associação de quatro extratos hidroalcoolicos obtidos de plantas brasileiras (Paullinia cupana, Trichilia catigua, Ptychopetalum olacoides e Zingiber officinale) com conhecida ação neuroprotetora, anti-inflamatória, antioxidante e antidepressiva. O bilobalide é um componente extraído das folhas do Ginkgo biloba, que tem comprovada ação neuroprotetora nos sistemas nervosos central e periférico. O presente estudo avaliou os efeitos da Catuama ${ }^{\circledR}$ e do bilobalide na regeneração nervosa periférica de ratos submetidos à seç̧ão do nervo isquiático. Foram utilizados 40 ratos com implante de tubo de silicone preenchido por colágeno líquido, deixando-se um intervalo entre os segmentos nervosos de $10 \mathrm{~mm}$. Os animais foram divididos em 4 grupos: o grupo controle (A); os grupos que receberam a Catuama ${ }^{\circledR}$ administrada por via oral nos primeiros 28 dias de pós-operatório, nas doses de 100 (B) e $400 \mathrm{mg} . \mathrm{kg}^{-1}$ (C); e o grupo que recebeu o bilobalide na dose de $200 \mu M(D)$, este, adicionado ao colágeno líquido utilizado no implante de silicone. Os animais foram avaliados na primeira, quinta e décima semanas de pós-operatório pelo teste de marcha. Na décima semana, foi realizada avaliação eletrofisiológica e análises quantitativa e qualitativa dos cortes histológicos de amostras do nervo isquiático e do músculo gastrocnêmio. Em todas as análises utilizadas observou-se excelente regeneração dos nervos, no entanto, não foi encontrada diferença significativa $(P>0,05)$ entre os grupos experimentais e controle.
\end{abstract}

Palavras-chave: reparação nervosa periférica, tubo de silicone, colágeno, fitoterapia.

\section{ABSTRACT}

The Catuama ${ }^{\circledR}$ is composed of four Brazilian plants extracts (Paullinia cupana, Trichilia catigua, Ptychopetalum olacoides e Zingiber officinale). The Catuama ${ }^{\circledR}$ is known as having neuroprotector, anti-inflammatory, antioxidant and antidepressant effects. Bilobalide, extracted from leaves of Ginkgo biloba, is known by its neuroprotective effect in the central and peripheral nervous systems. The present study evaluates the effect of Catuama ${ }^{\circledR}$ and bilobalide on peripheral nerve regeneration in rats following a sciatic nerve section. Sciatic nerve of forty adult rats was transected with a 10-mm gap and the proximal and distal nerve stumps were fixed in a silicone tube filled with liquid collagen. The animals were divided into four groups: the control group (A), two groups treated with Catuama $a^{\circledR}$ by gavage along 28 days after the surgery in different doses of 100 (B) and $400 \mathrm{mg}$. $\mathrm{kg}^{-1}(C)$ and the group using $200 \mu \mathrm{M}$ bilobalide (D) associated with the liquid collagen in the silicone tube. Evaluations were done by a walk test on the first, fifth and tenth week after the surgery. Electrophysiological stimulation and quantitative and qualitative histological analyses of the sciatic nerve and gastrocnemius muscle were also performed on the tenth week after the surgery. All groups showed good regeneration but no statistical difference was found between treatments and control groups $(P>0.05)$.

Key words: peripheral nerve repair, silicone tube, collagen, phytotherapy.

\section{INTRODUÇÃO}

Diferentes substâncias neuroestimulantes têm sido usadas para investigar o crescimento axonal entre os segmentos nervosos após secção transversal. Essas substâncias incluem o fator de crescimento nervoso (NGF), fator neurotrófico derivado do

IDepartamento de Medicina Veterinária, Universidade Federal de Lavras (UFLA), CP 3037, 37200-000, Lavras, MG, Brasil. E-mail: rodrigonorbertopereira@gmail.com. Autor para correspondência.

IIProfissional autônoma, Jaboticabal, SP, Brasil.

IIICentro de Ciências Agrárias, Ambientais e Biológicas (CCAAB), Universidade Federal do Recôncavo da Bahia (UFRB), Cruz das Almas, BA, Brasil.

IV Programa de Pós-graduação em Medicina Veterinária de Pequenos Animais, Universidade de Franca (Unifran), Franca, SP, Brasil.

vPrograma de Pós-graduação em Ciência Animal, CCA, UFPB, Areia, PB, Brasil.

${ }^{V I}$ Departamento de Patologia Veterinária, Faculdade de Ciências Agrárias e Veterinárias (FCAV), Universidade Estadual Paulista (UNESP), Jaboticabal, SP, Brasil. 
encéfalo (BDNF), colágeno, gel com laminina e outros (CHEN et al., 2004).

A Catuama ${ }^{\circledR}$ é a associação de quatro extratos hidroalcoolicos obtidos das seguintes plantas brasileiras: Paullinia cupana (guaraná; Sapindaceae) 40,31\%; Trichilia catigua (catuaba; Meliaceae) 28,23\%; Ptychopetalum olacoides (muirapuama; Olacaceae) 28,23\%; e Zingiber officinale (gengibre; Zingiberaceae) $3,26 \%$ (CAMPOS et al., 2004; OLIVEIRA et al., 2005). A Catuama ${ }^{\circledR}$, administrada oralmente a ratos por longos períodos, interferiu nos níveis de serotonina e dopamina, sugerindo-se que pode ser usada clinicamente em estágios de depressão leve e moderada, sozinha ou em associação com fármacos antidepressivos tradicionais (CAMPOS et al., 2004). PONTIERI et al. (2007) observaram que a Catuama ${ }^{\circledR}$ reverteu a fibrilação ventricular, prevenindo sua reindução e prolongando a condução intraventricular quando testada em coração de coelhos. Por outro lado, parece haver segurança no uso prolongado desse medicamento, tanto é que OLIVEIRA et al. (2005) não encontraram reações adversas severas ou mudanças hematológicas ou bioquímicas em pacientes humanos que receberam a administração crônica de $25 \mathrm{~mL}$ de Catuama $^{\circledR}$ duas vezes ao dia, por 28 dias.

O bilobalide, extraído das folhas do Ginkgo

biloba, apresenta múltiplos mecanismos de ação associados à neuroproteção, incluindo preservação da síntese de ATP mitocondrial e inibição de danos induzidos por apoptose e deterioração de membranas, induzida por isquemia no cérebro (DEFEUDIS, 2002; CHANDRASEKARAN et al., 2003). O bilobalide também tem sido utilizado com sucesso na reparação nervosa periférica por CHEN et al. (2004). Esses autores mostraram que o bilobalide apresentou alta taxa de formação de cabo de regeneração e maior número de axônios mielinizados, quando comparados ao controle. Assim, o presente estudo teve por objetivo verificar, funcional e morfologimente, a ação sistêmica da Catuama ${ }^{\circledR}$ administrada via oral e a ação local do bilobalide sobre a regeneração do nervo isquiático de ratos após transecção completa.

\section{MATERIAL E MÉTODOS}

Foram utilizados 40 ratos Wistar (Rattus norvegicus), machos, clinicamente sadios, pesando entre 250 e $350 \mathrm{~g}$. Os animais foram acomodados em gaiolas individuais, em ambiente controlado à temperatura de $22^{\circ} \mathrm{C}$, em ciclos diários de $12 \mathrm{~h}$ de luz. Água e ração comercial foram oferecidas ad libitum.

Para a realização da neurotomia, os animais foram anestesiados com associação de cetamina (75mg.kg-1 IM) e xilazina (10mg.kg-1 I.M.), segundo OLIVEIRA, (2010). Após antissepsia, foi realizada a incisão de pele na face lateral da coxa direita. Fáscias e grupos musculares foram separados por dissecção romba. Após a exposição do nervo isquiático direito, foi realizada sua transecção completa. Em seguida, os segmentos nervosos, proximal e distal, foram introduzidos e fixados ao tubo de silicone com ponto único em " $U$ " ao epineuro utilizando náilon 9-0, deixando um espaço de $10 \mathrm{~mm}$ entre os segmentos. O espaço entre os segmentos foi preenchido com solução contendo uma parte de colágeno em fase líquida (Vitrogen $3 \mathrm{mg} \mathrm{ml}^{-1}$ ) para uma parte de solução fisiológica com o auxílio de agulha $26 \mathrm{G}$, inserida no lúmen da câmara de silicone, evitando a formação de bolhas. Esses procedimentos foram baseados em DELISTOIANOV et. al. (2008). A camada muscular foi reaproximada com fio de náilon 4-0 e a sutura de pele em pontos simples separados com fio de náilon 2-0 (CHEN et al., 2004; HSU et al., 2004).

Os animais foram divididos em quatro grupos, com dez animais cada. Nos grupos A e D, os animais receberam solução salina via oral $\left(10 \mathrm{ml} . \mathrm{kg}^{-1}\right)$ nos primeiros 28 dias após a cirurgia. Nos grupos B e C, os animais foram tratados, respectivamente, com 100mg. $\mathrm{kg}^{-1}$ e $400 \mathrm{mg} . \mathrm{kg}^{-1}$ de Catuama ${ }^{\circledR}$ (Laboratório Catarinense, Joinville, SC, Brasil) nos primeiros 28 dias de pós-operatório (VAZ et al., 1997; CAMPOS et al., 2004; OLIVEIRA et al., 2005). Nos animais do grupo D, foram adicionados $200 \mu \mathrm{M}$ de bilobalide (Sigma) à solução de colágeno (Vitrogen) (CHEN et al., 2004).

A avaliação funcional foi realizada no préoperatório, na primeira, quinta e décima semanas de pós-operatório, por meio da análise do teste de marcha, introduzido por DE MEDINACELI et al. (1982), segundo ABREU et al. (2002). Na décima semana do pós-operatório todos os animais foram submetidos à anestesia para exposição dos nervos no local das intervenções cirúrgicas. Para avaliar a recuperação motora, foram realizadas, com eletroestimulador, modelo AP585 (VMV Biotherapy), estimulações dos segmentos nervosos proximais e distais e do cabo de regeneração formado entre os segmentos nervosos, conforme descrito por DELISTOIANOV et al. (2008). Após o exame eletrofisiológico, foi realizada a eutanásia dos animais, seguindo-se remoção das porções dos nervos isquiáticos do local do implante com tubo de silicone, com as margens $(5 \mathrm{~mm})$ proximal e distal do nervo. Fragmentos dos músculos gastrocnêmios direito e esquerdo foram colhidos e fixados em formalina a $10 \%$, tamponada com fosfatos, $\mathrm{pH} 7,2$. Após período de fixação, foram 
incluídos em parafina e obtidos cortes transversais, que foram corados com Hematoxilina e Eosina (HE) e tricrômico de Masson (TM), segundo rotina do laboratório. As amostras foram avaliadas em microscópio de luz. As alterações morfológicas dos músculos gastrocnêmios foram utilizadas para determinar o grau de atrofia muscular em escala de I a III, correspondendo à atrofia muscular leve, moderada e severa.

As amostras dos nervos foram igualmente fixadas e processadas para inclusão em parafina, obtendo-se cortes transversais de $3 \mu \mathrm{m}$ de espessura, também corados pelo HE e TM. Amostras semelhantes também foram fixadas em glutaraldeído, pós-fixadas em tetróxido de ósmio e incluídas em historesina a base de metacrilato, cortadas com $1 \mu \mathrm{m}$ de espessura, coradas com azul de toluidina (AT) e avaliadas em microscópio de luz.

Os cortes transversais dos segmentos nervosos foram avaliados quanto à presença de axônios mielinizados, proliferação axonal, infiltrado de células inflamatórias e degeneração walleriana. Os cortes transversais dos cabos de regeneração formados entre os segmentos nervosos foram avaliados quanto à área e contagem de axônios mielinizados, utilizando o microscópio Leica DM 5000B com o software Leica Application Suite (LAS) 2.5.1R1.

A análise estatística foi realizada utilizando o software SAS (Statistical Analisys System), aplicando-se o teste de Tukey para dados paramétricos e o teste de Kruskal-Wallis para os dados não paramétricos. O nível de significância foi estabelecido em $\mathrm{P}<0,05$.

\section{RESULTADOS E DISCUSSÃO}

Foram excluídos da avaliação do teste de marcha 16 animais: 14 indivíduos que apresentaram amputação total ou parcial de um dos dígitos $(35 \%$ do total), e 2 animais ( $5 \%$ do total), que apresentaram lesões no membro não operado, provavelmente, devido à aplicação intramuscular da anestesia, realizada na face caudal da coxa esquerda. $\mathrm{O}$ índice de função do nervo isquiático (IFI) variou entre os tempos observados, mantendo o mesmo padrão de distribuição entre os grupos, não havendo diferença entre eles, segundo a tabela 1 , exceto na semana 0 , antes dos animais serem submetidos ao procedimento cirúrgico. Os cabos de regeneração (CR) formados apresentavam diâmetro visivelmente inferior aos segmentos nervosos de origem. Houve formação de cabo de regeneração em todos os animais do grupo $\mathrm{B}$, enquanto $80 \%$ dos animais formaram cabo de regeneração no grupo controle, todavia essa diferença não foi significativa (tabela 2).

Os resultados da avaliação da resposta de contração muscular à eletroestimulação (tabela 2) foram positivos em todos os animais que apresentaram cabo de regeneração, exceto em um animal do grupo A (controle), que apresentou cabo de regeneração não responsivo à estimulação elétrica. A resposta à estimulação elétrica não apresentou diferença entre os grupos experimentais. A presença de resposta positiva em todos os animais que formaram cabo de regeneração, exceto um, demonstrou que, na décima semana de pós-operatório, o cabo de regeneração formado pelo segmento nervoso proximal foi capaz de alcançar seu órgão alvo e transmitir o estímulo elétrico, levando à contração muscular. Os cabos de regeneração formados foram avaliados em relação as suas áreas em corte transversal, não demonstrando diferença $(\mathrm{P}<0,05)$ entre os grupos (Tabela 2$)$.

Pela análise histológica dos cabos de regeneração, notou-se a presença de grande quantidade de axônios, axônios mielinizados e em processo de mielinização. Presença de grande

Tabela 1 - Médias dos valores do índice de função do isquiático (IFI) de ratos Wistar (Rattus norvegicus), submetidos à secção de nervo isquiático, seguida da reparação com tubo de silicone preenchido por colágeno líquido e tratados com solução fisiológica (grupo $\mathrm{A}$, controle), Catuama ${ }^{\circledR}$ nas doses de $100 \mathrm{mg} \mathrm{kg}^{-1}$ (grupo B) e $400 \mathrm{mg} \mathrm{kg}^{-1}$ (grupo C) e bilobalide na dose de $200 \mu \mathrm{M}$ (grupo D).

\begin{tabular}{lllll}
\hline Semana & \multicolumn{1}{c}{ A } & B & C & D \\
\hline 0 & $-0,501^{\mathrm{aA}}$ & $-9,599^{\mathrm{aAB}}$ & $-6,216^{\mathrm{aAB}}$ & $-20,827^{\mathrm{aB}}$ \\
1 & $-48,422^{\mathrm{bA}}$ & $-53,023^{\mathrm{bA}}$ & $-54,495^{\mathrm{bA}}$ & $-64,769^{\mathrm{bA}}$ \\
5 & $-69,089^{\mathrm{bA}}$ & $-78,299^{\mathrm{cA}}$ & $-67,892^{\mathrm{bA}}$ & $-80,596^{\mathrm{bA}}$ \\
10 & $-68,498^{\mathrm{bA}}$ & $-65,35^{\mathrm{bcA}}$ & $-68,777^{\mathrm{bA}}$ & $-65,941^{\mathrm{bA}}$ \\
\hline
\end{tabular}

Médias seguidas de letras minúsculas distintas na mesma coluna e maiúsculas distintas na mesma linha diferem entre si pelo teste Tukey $(\mathrm{P}<0,05)$. A: grupo controle, B: grupo Catuama ${ }^{\circledR} 100 \mathrm{mg}, \mathrm{C}$ : grupo Catuama ${ }^{\circledR} 400 \mathrm{mg}$ e D: grupo bilobalide $200 \mu \mathrm{M}$. 
Tabela 2 - Número de axônios por área em $\mu^{2}$ (NA), presença de cabo de regeneração (CR), eletroestimulação positiva (EE), área do cabo de regeneração (ACR), grau de degeneração do segmento distal (GDSD) e grau de atrofia do músculo gastrocnêmio do membro operado (GAM) de ratos Wistar (Rattus norvegicus), submetidos à secção de nervo isquiático, seguida da reparação com tubo de silicone preenchido por colágeno líquido e tratados com solução físiológica (grupo A, controle), Catuama ${ }^{\circledR}$ na dose de $100 \mathrm{mg} \mathrm{kg}^{-1}$ (grupo B) e $400 \mathrm{mg} \mathrm{kg}^{-1}$ (grupo C) e bilobalide na dose de $200 \mu \mathrm{M}$ (grupo D).

\begin{tabular}{|c|c|c|c|c|c|}
\hline Parâmetro & A & B & $\mathrm{C}$ & D & $\mathrm{CV}(\%)$ \\
\hline $\mathrm{NA}^{1}$ & 0,0546 & 0,0523 & 0,0598 & 0,0629 & 19,15 \\
\hline $\mathrm{CR}(\%)^{*}$ & 80 & 100 & 88,89 & 90 & - \\
\hline $\mathrm{EE}(\%) *$ & 70 & 100 & 88,89 & 90 & - \\
\hline $\operatorname{ACR}^{1}\left(\mu m^{2}\right)$ & 256558 & 274068 & 223276 & 252259 & 3,43 \\
\hline GDSD & $2,7^{\mathrm{a}}$ & $1,5^{\mathrm{a}}$ & $2,0^{\mathrm{a}}$ & $2,1^{\mathrm{a}}$ & - \\
\hline GAM & $2,2^{\mathrm{a}}$ & $2,0^{\mathrm{a}}$ & $2,1^{\mathrm{a}}$ & $2,3^{\mathrm{a}}$ & - \\
\hline
\end{tabular}

${ }^{1}$ Não houve diferença significativa pelo este Tukey $(\mathrm{P}>0,05)$. A: grupo controle, B: grupo Catuama ${ }^{\circledR} 100 \mathrm{mg}$, C: grupo Catuama ${ }^{\circledR} 400 \mathrm{mg}$; e D: grupo bilobalide $200 \mu \mathrm{M}$. CV: coeficiente de variação. * Valores referentes à percentagem de presença de CR ou EE.

quantidade de vasos sanguíneos e hemossiderina (Figura 1A e B). Esse pigmento é derivado do metabolismo de ferro e é um indicativo de hemorragia pós-secção nervosa. Quanto à proliferação axonal, os cabos de regeneração foram avaliados pela contagem do número de axônios mielinizados por área $\left(\mu \mathrm{m}^{2}\right)$. Os resultados estão apresentados na tabela 2 e indicam não haver diferença $(\mathrm{P}<0,05)$ entre os grupos experimentais. A presença de axônios mielinizados (Figura 1E e F) é um indício histológico de reparação nervosa periférica e está de acordo com os achados da avaliação funcional e avaliação eletrofisiológica, demonstrando que os cabos de regeneração formados foram capazes de alcançar seus órgãos-alvo e transmitir estímulo elétrico, causando contração muscular e melhorando a qualidade da pegada.

Os segmentos nervosos proximais apresentaram à microscopia de luz, em corte transversal, aspecto típico de um nervo periférico, constituído por vários fascículos, cada qual com muitas fibras nervosas. O epineuro do feixe nervoso e o perineuro de cada fascículo apresentaram-se característicos. Observaram-se ainda, fibras nervosas em espessuras diferentes, com revestimento mielínico e morfologia muito próxima do normal. A mielina ficou muito bem evidenciada na cor vermelha pelo TM.

Os segmentos nervosos distais foram avaliados em relação ao grau de degeneração nervosa (GDSD), em uma escala de I a IV. A diferença foi nítida entre os animais que não apresentaram cabo de regeneração (Figura 1C) e os animais em que houve a formação do cabo (Figura 1D). Não houve diferença $(\mathrm{P}<0,05)$ entre os grupos em relação ao GDSD, entretanto, o grupo B apresentou a menor média de GDSD, seguido dos grupos $\mathrm{Ce} D$, que também tiveram desempenho superior ao grupo controle (Tabela 2). O único animal que formou cabo de regeneração entre os segmentos nervosos e teve resposta negativa ao estímulo elétrico, apresentou grande quantidade de tecido conjuntivo e poucos axônios mielinizados no cabo de regeneração. Apresentou, ainda, grau IV de degeneração do segmento distal, semelhante aos animais que não formaram cabo de regeneração. A atrofia do músculo gastrocnêmio, diretamente relacionada ao processo de desnervação do músculo pelo nervo isquiático, apresentou-se mais acentuada nos animais que não formaram cabo de regeneração (Figura 1G), quando comparada aos animais que formaram cabo de regeneração e tinham resposta positiva de contração muscular à eletroestimulação. Todavia, não houve diferença $(\mathrm{P}<0,05)$ com relação ao grau de atrofia muscular entre os grupos experimentais.

A regeneração nervosa periférica é um assunto amplamente estudado por diversos grupos de pesquisa em todo o mundo. A recuperação funcional de um nervo lesado continua sendo um desafio clínico em diferentes espécies. A hipótese avaliada foi a potencial ação neuroprotetora da Catuama ${ }^{\circledR}$ no SNP e sua interferência no processo de regeneração nervosa periférica. Os achados encontrados na avaliação pelo teste da função do nervo isquiático foram compatíveis com os encontrados por MONTE-RASO et al. (2008). Nas primeiras semanas de avaliação, as pegadas analisadas tinham qualidade insatisfatória, entretanto, nas avaliações seguintes, a análise das pegadas foi realizada com mais facilidade. Todas as avaliações foram realizadas pelo mesmo examinador, que não conhecia a origem dos grupos. Os achados do teste de função do nervo isquiático foram compatíveis 




Figura 1 - Fotomicrografias de tecido nervoso e muscular de ratos Wistar (Rattus norvegicus) submetidos à secção de nervo isquiático, seguida da reparação com tubo de silicone, preenchido por colágeno líquido e tratados com solução fisiológica (grupo controle), Catuama ${ }^{\circledR}$ nas doses de $100 \mathrm{mg}$ kg-1 (grupo Catuama 100mg) e 400mg kg-1 (grupo Catuama 400mg) e bilobalide na dose de $200 \mu \mathrm{M}$ (grupo bilobalide) à décima semana de pósoperatório. A e B: corte transversal do cabo de regeneração formado entre os segmentos nervosos de rato do grupo controle, A, e do grupo bilobalide, B. Presença de vasos sanguíneos (setas), hemossiderina (pontas de setas). Hematoxilina e Eosina, barra: $20 \mu \mathrm{m}$. C: corte transversal do segmento distal a secção de nervo isquiático de rato do grupo controle que não apresentou formação de cabo de regeneração. Degeneração walleriana severa (grau IV) com presença de câmara de digestão (seta), vacúolos (pontas de setas), ausência de axônios mielinizados e áreas de fibrose (asterisco). Tricrômico de Masson, barra: $100 \mu \mathrm{m}$. D: corte transversal do segmento distal a seç̧ão de nervo isquiático de um rato do grupo Catuama $100 \mathrm{mg}$ com formação de cabo de regeneração. Presença de axônios mielinizados (setas) e discreta degeneração walleriana (grau I). Tricrômico de Masson, barra: $50 \mu \mathrm{m}$. E e F: corte transversal do cabo de regeneração de nervo isquiático de rato do grupo controle, E, e do grupo Catuama 400mg, F. Presença de axônios mielinizados em grande quantidade e com diâmetros diferentes (ponta de setas). Azul de toluidina, barra: $20 \mu \mathrm{m}$. G: corte transversal do músculo gastrocnêmio de rato do grupo controle que não apresentou formação de cabo de regeneração. Presença de atrofia muscular severa com diminuição do tamanho das fibras musculares que têm perfis angulares e alongados e áreas com agrupamento de núcleos e macrófagos (asteriscos). H: corte transversal do músculo gastrocnêmio do membro não operado do mesmo animal de G. Presença de fibras musculares de perfil normal e núcleos periféricos (ponta de setas).

Ciência Rural, v.44, n.5, mai, 2014. 
com os achados histológicos, histomorfométricos e eletrofisiológicos, indicando viabilidade de execução do experimento e fidedignidade de resultados.

A avaliação eletrofisiológica, segundo a técnica de DELISTOIANOV et al. (2008), foi adequada para a avaliação dos efeitos da catuama e do bilobalide na regeneração nervosa periférica, apesar de ser uma análise qualitativa, dando como resultado apenas positivo ou negativo. Entretanto, LABRADOR et al. (1998) e de MURAKAMI et al. (2003), utilizando mensuração do potencial evocado, realizaram uma análise quantitativa da capacidade de condutividade elétrica do nervo. A análise das biopsias nervosas demonstrou que o período de 10 semanas foi suficiente para a formação de um cabo de regeneração com presença de grande quantidade de axônios mielinizados e em processo de mielinização. Esses achados foram compatíveis com o retorno da capacidade de transmissão do estímulo elétrico e contração muscular e com a melhora da qualidade da pegada no teste de função do nervo isquiático, observado em todos os grupos experimentais.

$\mathrm{O}$ grupo bilobalide apresentou uma porcentagem de formação de cabo de regeneração de $88,89 \%$, não apresentando diferença em relação ao controle, que obteve $80 \%$ de formação. CHEN et al. (2004) obtiveram $50 \%$ de formação de cabo de regeneração quando utilizaram a mesma dose de $200 \mu \mathrm{g}$, mas com um intervalo entre os segmentos nervosos de $15 \mathrm{~mm}$, num período de oito semanas. No experimento citado, a porcentagem de formação do cabo de regeneração do grupo controle foi de apenas $10 \%$. O tamanho do intervalo entre os segmentos nervosos e o tempo de observação pósoperatória pode ter sido responsável pela divergência de resultados encontrada entre os experimentos. Talvez o efeito benéfico do bilobalide tenha ficado evidente no modelo de CHEN et al. (2004), por haver um intervalo maior entre os segmentos nervosos e, portanto, em condições de maior adversidade à regeneração. Assim, a hipótese da Catuama ${ }^{\circledR}$ e seus componentes que tem ação no sistema nervoso central (CAMPOS et al., 2004; OLIVEIRA et al., 2005) terem ação sobre a regeneração nervosa periférica, talvez, possa ser demonstrada por modelos experimentais que aumentem o desafio à reparação nervosa periférica por meio de intervalos mais longos entre os segmentos nervosos.

A Catuama ${ }^{\circledR}$ e o bilobalide podem ter apresentado algum efeito nas fases iniciais da reparação nervosa durante as primeiras semanas de pós-operatório. Todavia, esse possível efeito só foi analisado pela avaliação funcional, que apresenta baixa confiabilidade, como demonstrado por MONTE-RASO et al. (2008), justamente nas primeiras semanas após a cirurgia. Uma proposta para a avaliação deste possível efeito seria aumentar os grupos experimentais, fazendo a análise histológica e histomorfométrica destes novos grupos durante as primeiras semanas de pós-operatório.

\section{CONCLUSÃO}

A regeneração do nervo isquiático em câmara de silicone, acrescido de colágeno líquido, pode ser considerada satisfatória, servindo de subsídio para outros estudos. Por outro lado, a Catuama ${ }^{\circledR}$ nas doses avaliadas e o bilobalide não demonstraram efeitos significativos sobre a regeneração do nervo, tanto em termos funcionais quanto estruturais.

\section{AGRADECIMENTO}

À Fundação de Amparo à Pesquisa do Estado de São Paulo (FAPESP), pela concessão de recursos.

\section{COMITÊ DE ÉTICA E BIOSSEGURANÇA}

O trabalho foi aprovado pela Comissão de Ética e Bem Estar Animal da Faculdade de Ciências Agrárias e Veterinárias da Universidade Estadual Paulista "Júlio de Mesquita Filho", protocolo no. 021338-06, e os estudos em animais foram realizados de acordo com normas éticas.

\section{REFERÊNCIAS}

ABREU, A.A.N. et al. Avaliação dos gangliosídios na regeneração nervosa em ratos. Revista Brasileira de Ortopedia, v.37, n.1/2, p.3944, 2002. Disponível em: <http://www.rbo.org.br/Desktopdefault.asp x? tabid=132\&ItemID=729\&edicaoid=94>. Acesso em: 25 nov. 2013.

CAMPOS, M.M. et al. Pharmacological and neurochemical evidence for antidepressant-like effects of the herbal product Catuama. Pharmacology, Biochemistry and Behavior, v.78, p.757-764, 2004. Disponível em: <http://www.sciencedirect.com/ science/article/pii/S0091305704001893>. Acesso em: 25 nov. 2013. Doi:10.1016/j.pbb.2004.05.024.

CHANDRASEKARAN, K. et al. Neuroprotective effects of bilobalide, a component of Ginkgo biloba extract (EGb 761) in global brain ischemia and excitotoxicity-induced neuronal death. Pharmacopsychiatry, v.36, supl.1, p.S89-94, 2003. Disponível em: <https://www.thiemeconnect.com/DOI/DOI?10.1055/s-2003-40447>. Acesso em: 25 nov. 2013; doi: 10.1055/s-2003-40447.

CHEN, Y.S. et al. Effect of bilobalide on peripheral nerve regeneration. Biomaterials, v.25, p.509-514, 2004. Disponível em: <http://www. sciencedirect.com/science/article/pii/S0142961203005489>. Acesso em: 25 nov. 2013. Doi: 10.1016/S0142-9612(03)00548-9.

DE MEDINACELI, L. et al. An index of the functional condition of rat sciatic nerve based on measurements made from walking tracks. Experimental Neurology, v.77, p.634-643, 1982. Disponível em: <http://www.sciencedirect.com/science/ 
article/pii/0014488682902345>. Acesso em: 25 nov. 2013. Doi: 10.1016/0014-4886(82)90234-5.

DEFEUDIS, F.V. Bilobalide and neuroprotection. Pharmacology Research, v.46, n.6, p.565-568, 2002. Disponível em: <http:// www.sciencedirect.com/science/article/pii/S1043661802002335>. Acesso em: 25 nov. 2013. Doi: 10.1016/S1043-6618(02)00233-5.

DELISTOIANOV, N. et al. Implante de tubo de silicone com e sem colágeno na regeneração de nervos de eqüinos. Ciência Rural, v.38, n.6, p.1667-1674 2008. Disponível em: <http://www.scielo.br/ scielo.php?pid=S0103-84782008000600027\&script=sci_arttext $>$. Acesso em: nov. 2013. Doi: 10.1590/S0103-84782008000600027.

HSU, S.H. et al. In vitro and in vivo effects of Ginkgo biloba extract Egb 761 on seeded Schwann cells within poly (DL-lactic acid-co-glycolic acid) conduits for peripheral nerve regeneration. Journal of Biomaterials Applications, v.19, n.2, p.163-182, 2004. Disponível em: <http://jba.sagepub.com/content/19/2/163.full pdf $>$. Acesso em: 25 nov. 2013. Doi: 10.1177/0885328204045580.

LABRADOR, R.O. et al. Influence of collagen and laminin gels concentration on nerve regeneration after resection and tube repair. Experimental Neurology, v.149, n.1, p.243-252, 1998. Disponível em: <http:/www.sciencedirect.com/science/article/pii/ S0014488697966504>. Acesso em: 25 nov. 2013. Doi: 10.1006 exnr.1997.6650.

MONTE-RASO, V.V. et al. Is the sciatic functional index reliable and reproducible. Journal of Neuroscience Methods, v.170 p.255-261, 2008. Disponível em: <http://www.sciencedirect.com/ science/article/pii/S0165027008000782>. Acesso em: nov. 2013. Doi: 10.1016/j.jneumeth.2008.01.022.
MURAKAMI, T. et al. Transplanted neuronal progenitor cells in a peripheral nerve gap promote nerve repair. Brain Research, v.974, n.1-2, p.17-24, 2003. Disponível em : <http://www.sciencedirect. com/science/article/pii/S0006899303025393>. Acesso em: nov. 2013. Doi: 10.1016/S0006-8993(03)02539-3.

OLIVEIRA, C.H. et al. Clinical toxicology study of the herbal medicinal extract of Paullinia cupana, Trichilia catigua, Ptychopetalum olacoides and Zingiber officinale $\left(\right.$ Catuama $\left.^{\circledR}\right)$ in healthy volunteers. Phytotherapy Research, v.19, n.1, p.54-57, 2005. Disponível em: <http://onlinelibrary.wiley.com/doi/10.1002/ ptr.1484/abstract;jsessionid=9313BD21E8E5685CE0AD9693C 7B73051.f02t02>. Acesso em: nov. 2013. Doi: 10.1002/ptr.1484.

OLIVEIRA, H.P. Protocolos anestésicos comumente utilizados em animais de pequeno porte. Belo Horizonte: Comitê de Ética em Experimentação Animal, UFMG. Online. Disponível em: $<$ http://www.ufmg.br/bioetica/cetea/index.php?option=com_conte nt\&task=view\&id=22\&Itemid=35>. Acesso em: 25 jan. 2010 .

PONTIERI, V. et al. The herbal drug Catuama reverts and prevents ventricular fibrillation in the isolated rabbit heart. Journal of Electrocardiology, v.40, p.534.e1-534.e8, 2007. Disponível em: $<$ http:// www.sciencedirect.com/science/article/pii/S0022073607006139>. Acesso em: nov. 2013. Doi: 10.1016/j.jelectrocard.2007.06.002.

VAZ, Z.R. et al. Analgesic effect of herbal medicine Catuama in thermal and chemical models of nociception in mice. Phytotherapy Research, v.11. n.2, p.101-106, 1997. Disponível em: <http:// onlinelibrary.wiley.com/doi/10.1002/\%28SICI\%2910991573\%28199703\%2911:2\%3C101::AID-PTR28\%3E3.0.CO;2-U/ abstract>. Acesso em: nov. 2013. Doi: 10.1002/(SICI)10991573(199703)11:2<101::AID-PTR28>3.0.CO;2-U 